\title{
Echocardiography-Based Selection of Quiescent Heart Phases
}

\section{Implications for Cardiac Imaging}

Srini Tridandapani, PhD, MD, J. Brian Fowlkes, PhD, Jonathan M. Rubin MD, PhD

\begin{abstract}
Objective. We compared the potential effectiveness of echocardiography with electrocardiography (ECG) for the selection of phases within the cardiac cycle during which there is relatively little motion. Determination of such phases may be important for triggering other modalities such as computed tomography and magnetic resonance imaging. Methods. In a preliminary study in 2 volunteers, simultaneous ECG and cardiac echocardiographic data were obtained and analyzed. Various phases of the echocardiographic data were cross-correlated from beat to beat. Time point pairs from different cardiac cycles with correlation of 0.8 were selected. Results. Beat-to-beat correlation values peaked at around $80 \%$ of the $R-R^{\prime}$ interval near the cardiac apex and around $30 \%$ of the $R-R^{\prime}$ interval near the cardiac base for 1 subject. For the other subject, the correlation values peaked at around $75 \%$ of the $R-R^{\prime}$ interval near the cardiac apex. Conclusions. The cardiac cycle phase having maximum spatial cross-correlation for the longest period varied from subject to subject and also on the portion of the heart that was being imaged. Echocardiography may be superior to ECG in determining points in the cardiac cycle when the heart is relatively stationary and can potentially provide more optimal triggering information for cardiac computed tomography or magnetic resonance imaging. Key words: cardiac computed tomographic angiography; cardiac gating; cardiac imaging; sonographic gating.
\end{abstract}

\section{Abbreviations}

CT, computed tomography; ECG, electrocardiography; $\mathrm{MRI}$, magnetic resonance imaging; 3D, 3-dimensional

Received May 10, 2005, from the Department of Radiology, University of Michigan, Ann Arbor, Michigan USA. Revision requested May 31, 2005. Revised manuscript accepted for publication June 26, 2005.

We thank Charles R. Meyer, PhD, for useful discussions and comments on an earlier version of the manuscript. Ultrasound equipment and software were provided by GE Healthcare. This research was funded in part by a University of Michigan biomedical technology development award and a Siemens Medical Solutions, Inc/Radiological Society of North America research resident grant.

Address correspondence to Srini Tridandapani, PhD, MD, Department of Radiology, University of Michigan, 1500 E Medical Center Dr, UH B1D 502, Ann Arbor, MI 48103 USA.

E-mail: srinit@med.umich.edu

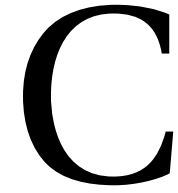

omputed tomography (CT) and magnetic resonance imaging (MRI) are increasingly being considered as potential alternatives to more invasive angiographic studies to evaluate the heart and, in particular, the coronary arteries. ${ }^{1,2}$ The fundamental challenge with cross-sectional imaging of the heart is that the heart is a moving target, and there is a need to acquire image slices at the same point in the cardiac cycle from beat to beat so that 3-dimensional (3D) volume reconstructions are optimally registered. Electrocardiography (ECG) is widely used to trigger image acquisition in these sophisticated imaging modalities. ${ }^{3}$ Although ECG is excellent at depicting the instantaneous electrical properties of the heart and is also very good at predicting the average spatial location of the heart, it is not necessarily capable of determining the instantaneous location of the 
heart from beat to beat. Echocardiography, a real-time instrument just like ECG, is able to determine the spatial properties of the heart and can, therefore, potentially be used as a trigger for slice acquisition in cardiac CT or MRI applications. ${ }^{4}$

In this preliminary work, our goal was to show, using 2-time cross-correlation of echocardiographic data from various time points within different cardiac cycles, that echocardiography is able to determine when the heart is quasistationary.

\section{Materials and Methods}

The human experiments for this research were performed after the study was approved by the Institutional Review Board for Medicine at University of Michigan. Prior written informed consent was obtained from the 2 subjects recruited for this study. Both subjects were healthy male volunteers (ages 57 and 38 years) without any arrhythmias or prior history of heart disease.

A System V/Vingmed sonographic scanner (GE Healthcare, Milwaukee, WI) was used to obtain the images. For each patient, M-mode images were obtained in the subxiphoid and left parasternal approaches with either a 2.5- or 5$\mathrm{MHz}$ phased array scan head. B-mode images were also obtained by these 2 approaches. For all the scanning sequences, simultaneous ECG data were also obtained with 2 leads: 1 connected to the right anterior chest at approximately the fourth intercostal space in the midclavicular line and the other connected to the left anterior chest at approximately the sixth intercostal space midaxillary line (lead II configuration). The images were stored on a computer disk and transferred to a workstation for later processing. The image sequences were processed by software routines written by us and by EchoMat (GE Healthcare) software routines for MATLAB (The MathWorks, Natick, MA).

We will describe our mathematical methods with a representative data sample obtained from 1 volunteer. In Figure 1A, we show a representative left parasternal B-mode image obtained near the apex from subject 1 . The M-mode and corresponding ECG signals are shown in Figure 1, B and $C$, respectively. The heart rate for this sample was 62 beats $/ \mathrm{min}$. The temporal resolution of the M-mode data for these sample data was 4 mil- liseconds; that is, nearly $250 \mathrm{M}$-mode lines were obtained for each heartbeat. The M-mode signal was range gated to the left ventricular wall for the analysis not only to reduce the computational complexity but also because of noise within the ventricular chamber. For this particular example, we choose the M-mode signal starting at a depth $d_{1}$ $\left(\approx 7.7 \mathrm{~cm}\right.$ in this case) and extending to a depth $d_{2}$ $(\approx 15.4 \mathrm{~cm}$ in this case). The ECG $\mathrm{R}$ wave was used to select the start of each cycle. Within each cycle, the range-gated $\mathrm{M}$-mode data corresponding to the depth range that we selected were used, and a correlation was performed between the M-mode signal in each 4-millisecond interval of that cycle and the M-mode signal in each 4-millisecond interval of a subsequent cycle. The cross-correlation was performed as follows:

$$
c\left(t_{1}, t_{2}\right)=\frac{\sum_{x=d_{1}}^{d_{2}}\left(X\left(x, t_{1}\right)-\bar{X}\left(t_{1}\right)\right)\left(X\left(x, t_{2}\right)-\bar{X}\left(t_{2}\right)\right)}{\sigma_{t_{1}} \sigma_{t_{2}}}
$$

where $c\left(t_{1}, t_{2}\right)$ was the 2-time spatial correlation between the 2 vectors of $M$-mode data at times $t_{1}$ (in the first cycle) and $t_{2}$ (in a subsequent cycle); $X(x, t)$ was the amplitude of the sonographic M-mode data obtained at time $t$ and depth $x ; \bar{X}(t)$ and $\sigma_{t}$ were the mean and SD, respectively, of the M-mode data vector at time $t$; and $d_{1}$ and $d_{2}$ represented the lower and upper limits of the depth range of the M-mode signal considered.

Once the cross-correlation values for all time point pairs from each cycle and a subsequent cycle were obtained, we could select those time point pairs with correlation values above some specified threshold.

\section{Results}

The correlation values obtained from the analysis described in "Materials and Methods" are plotted for the example case in Figure 1D. The x-axis in this plot represents time along the first cardiac cycle, and the y-axis represents time along a subsequent cardiac cycle. The color scale mapping of the correlation values is provided to the right of the graph: red represents time point pairs that are highly correlated, whereas dark blue represents time point pairs that are uncorrelated.

Note that the correlation peaks that are greater than 0.8 appear to be centered on the diagonal. This is intuitively reassuring and shows that, in the absence of an arrhythmia, ECG can be used, 


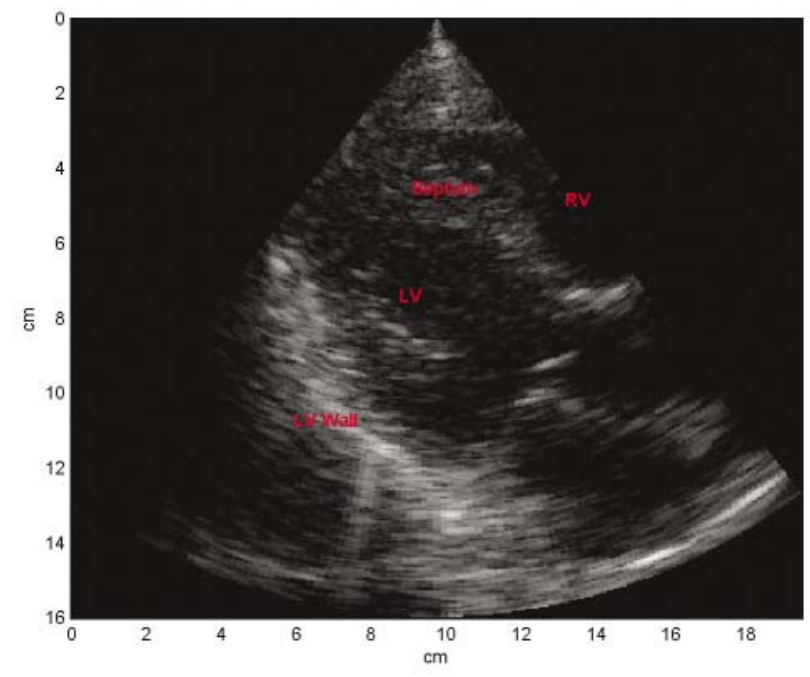

A

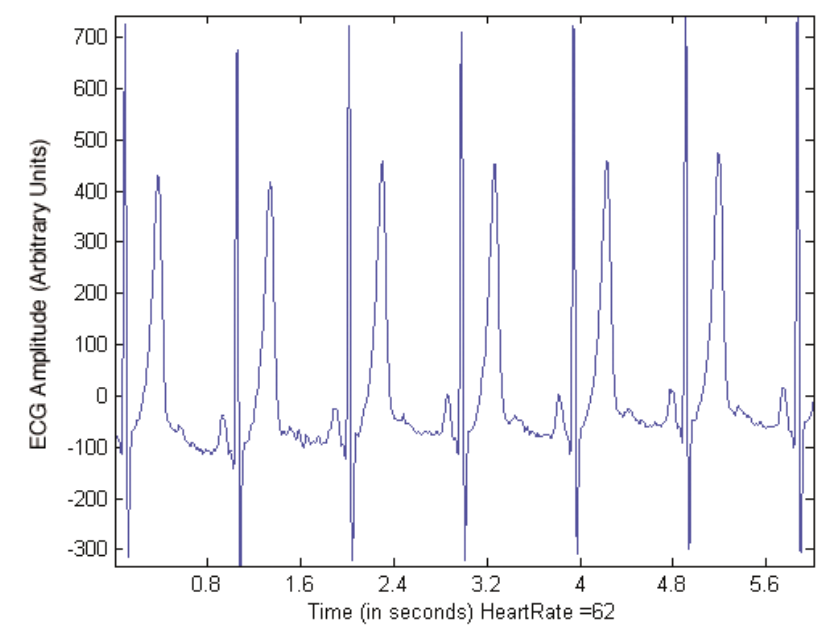

C

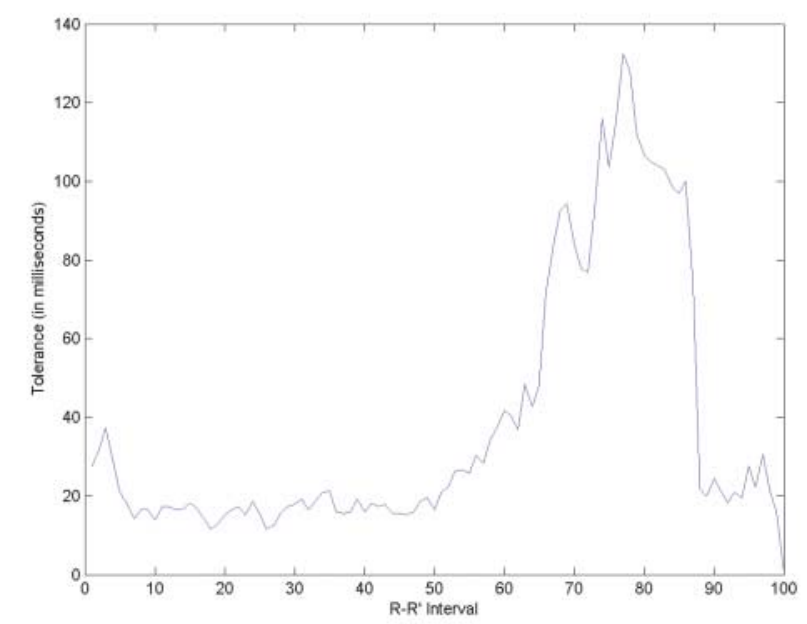

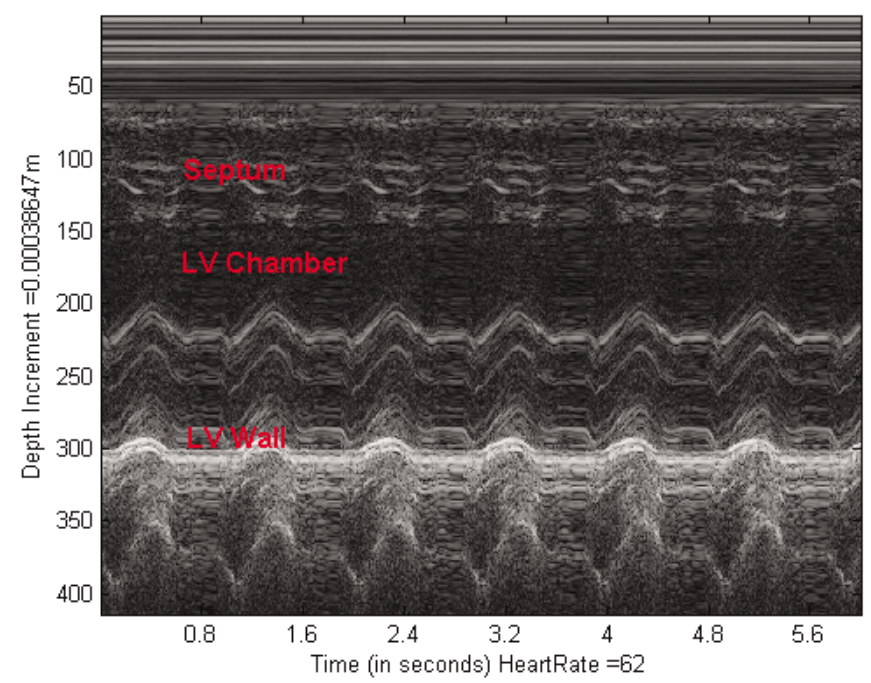

B

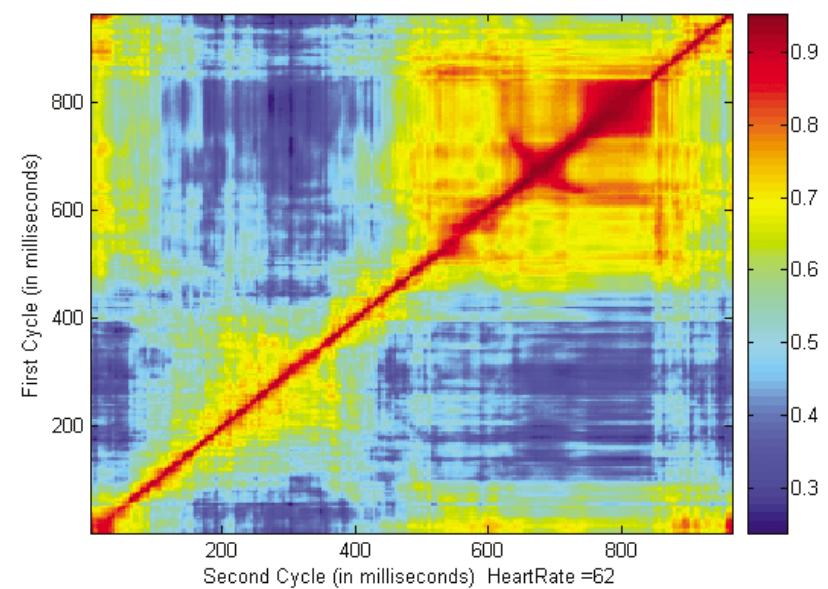

D

Figure 1. Subject 1 , data obtained in the long axis left parasternal view near the apex. A, B-mode image. B, Corresponding M-mode data. C, Corresponding ECG. D, Correlation plot of M-mode data selected over the far left ventricle wall between a randomly selected cycle and a subsequent cycle. The pixel color provides a measure of the correlation between time point pairs from 2 different cycles. The $x$-axis displays time along the second cycle, and the $y$-axis displays time along the first cycle. The color scale on the right provides the scale for the correlations. E, Tolerance values plotted as a percentage of the R-R' interval obtained by averaging correlations over 9 cardiac cycle pairs. Tolerance refers to the largest continuous block of time around the diagonal for which the correlation values are greater than 0.8. Note that the correlation values have a broad peak in the latter portion of the cardiac cycle. LV indicates left ventricle; and RV, right ventricle.

E 
for example, to trigger acquisition of a CT slice if CT scanners with infinitely fast gantry rotation speeds are available; however, the width of the correlation peak is not the same at every point along the diagonal. For example, at approximately 400 milliseconds, there is a correlation peak of greater than 0.8 corresponding also to 400 milliseconds on the y-axis. If a CT slice is triggered at this point, the slice would need to be completed in about 4 milliseconds (maximum temporal resolution for the $\mathrm{M}$ mode) because there is rapid decorrelation in the next few milliseconds. Later in the cycle, from approximately 740 to 840 milliseconds, we measured the correlation to be greater than 0.80 . Thus, for approximately $100 \mathrm{msec}$, there is very high correlation, which is enough time to obtain approximately half of a CT slice on most modern scanners.

An alternative method of looking at the correlation values is to consider the period that the correlation values remain above 0.8 for each $\mathrm{R}$ $\mathrm{R}^{\prime}$ interval. We define this as the "tolerance" or the quiescent period during which the heart is relatively stationary. In Figure 1E, we have plotted this tolerance as a percentage of the R$\mathrm{R}^{\prime}$ interval. The $\mathrm{x}$-axis shows the percent $\mathrm{R}-\mathrm{R}^{\prime}$ interval, and the $y$-axis shows the tolerance (quiescent period) obtained as an average from 9 cycles of correlation between each cycle and a subsequent cycle. For Figure 1E, we selected the largest segment of time points where the correlation continuously remained greater than 0.8 around the diagonal. Clearly, from Figure 1, D and E, the $65 \%$ to $85 \%$ interval is a good period to trigger the acquisition of a CT slice.

We now look at data obtained from a second subject, subject 2, in Figure 2. These data were also obtained in the long axis left parasternal view near the apex. Figure 2, A and B, shows the $\mathrm{B}$ - and the M-mode images, respectively. In this case, the mean heart rate was 58 beats $/ \mathrm{min}$; the corresponding ECG signal is shown in Figure 2C. The M-mode data from the far left ventricle wall were considered from a depth of $10.4 \mathrm{~cm}$ to a depth of $14.3 \mathrm{~cm}$, and correlations were performed as with the previous case. The correlation values are plotted in Figure 2D, and the tolerance values are shown in Figure 2E. We note here that the correlation peak is now around $75 \%$ to $85 \%$, and the peak is not as broad as it was with subject 1 .
Next, we look at data obtained again from subject 2, in Figure 3. These data were also obtained in the long axis left parasternal view but near the base. Figure 3, A and B, shows the B- and Mmode images, respectively. In this case, the mean heart rate was 56 beats/min; the corresponding ECG signal is shown in Figure 3C. The M-mode data from the left ventricle wall from a depth of $10.8 \mathrm{~cm}$ to a depth of $13.9 \mathrm{~cm}$ were considered, and correlations were performed. The correlation values are plotted in Figure 3D, and the tolerance values are shown in Figure 3E. We can see here that the correlation peaks appear earlier in the cardiac cycle between $20 \%$ and $40 \%$.

Finally, in Figure 4, we consolidate the tolerance (quiescent period) curves for the 3 cases. From this figure we conclude that the best time to trigger a CT scan would be slightly different between subjects 1 and 2 if the scan is to be obtained near the apex, although there is an overlapping time that would be satisfactory for both. We also conclude that the tolerance period varies considerably within a subject (in this case, subject 2) depending on the portion of the heart being evaluated.

\section{Discussion}

These preliminary results show that our technique can provide a noninvasive method of quantifying and tracking relative cardiac motion within a cardiac cycle; it can potentially be used to determine the optimal trigger time and, furthermore, the duration of the quiescent period in the cardiac cycle when CT slices could be obtained. Thus, even if ECG is to be used as a prospective trigger for technical reasons, information provided by our technique can be used to optimize this ECG trigger. Further development and refinement of this technique could obviate the need for radiation-intensive retrospective CT approaches for evaluating coronary arteries. (Of course, retrospective approaches can also provide functional information, such as ejection fraction, and can also be used to evaluate wall motion abnormalities.) It is clear that gating defined by cardiac position would be beneficial. In recent work using retrospective CT data, ${ }^{5}$ it was shown that Agatston scores ${ }^{6}$ and volumetric scores both proved to be highly dependent on the reconstruction interval used even with the most advanced CT scanners. It was also shown that the variable Agatston scores ${ }^{6}$ of diastolic 


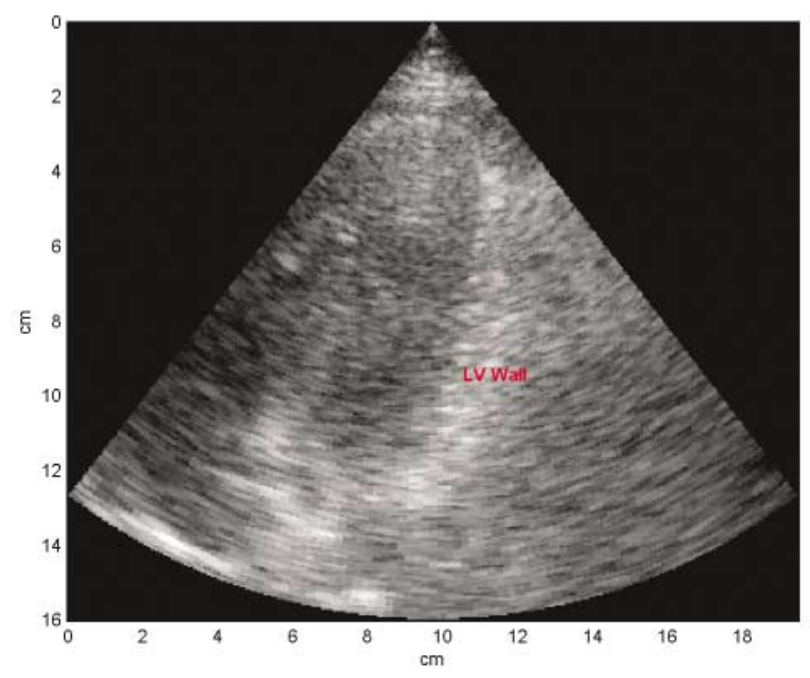

A

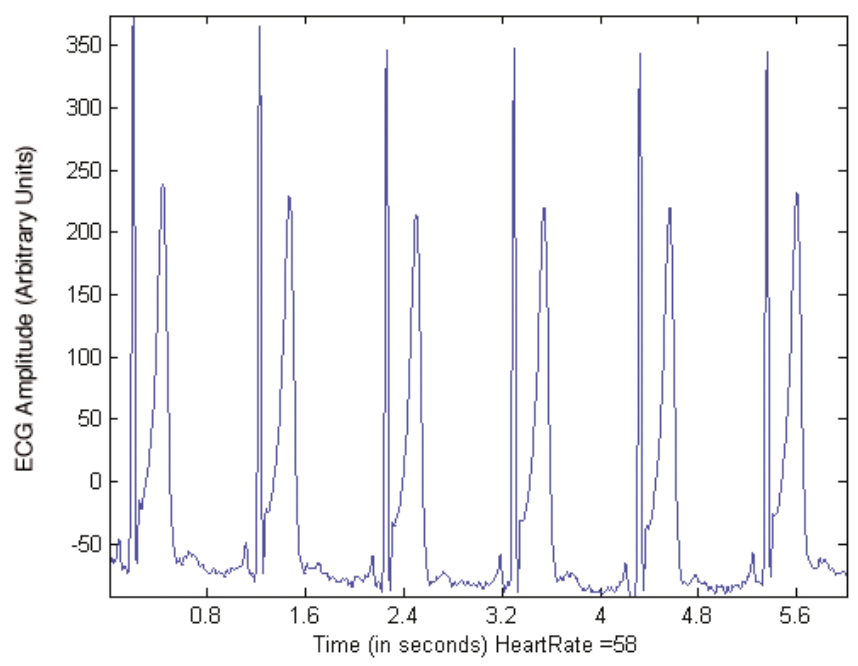

C

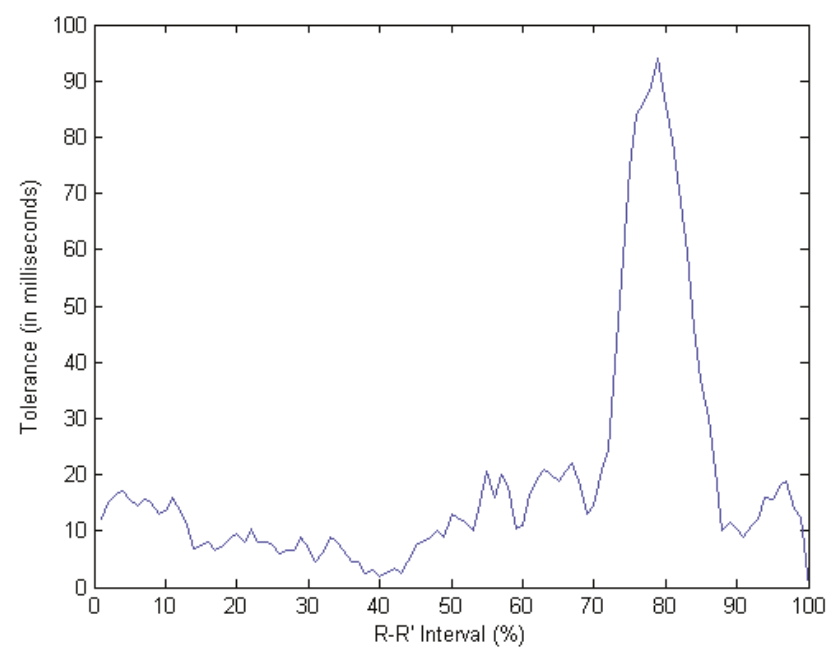

E

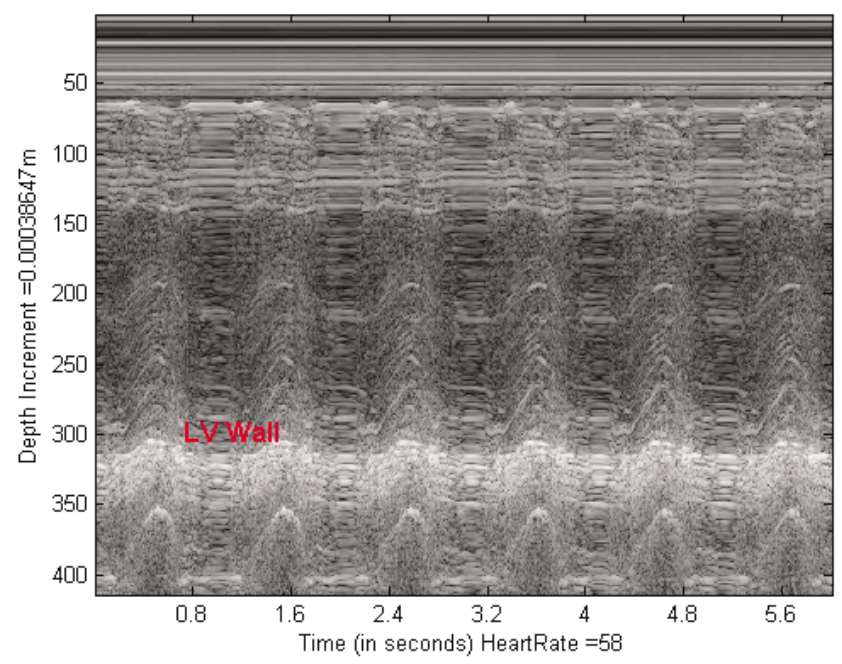

B

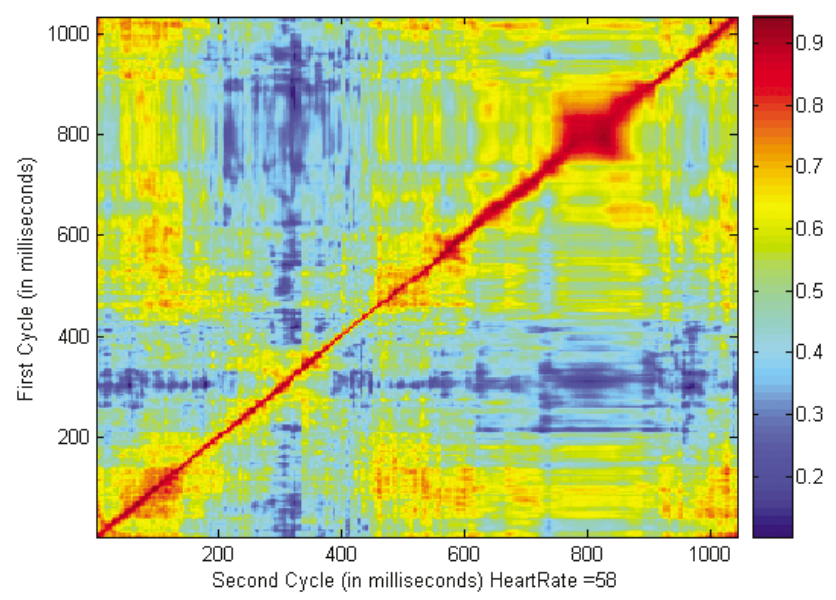

D

Figure 2. Subject 2, data obtained in the long axis left parasternal view near the apex. A, B-mode image. B, Corresponding M-mode data. C, Corresponding ECG. D, Correlation plot between a randomly selected cycle and a subsequent cycle. E, Tolerance values plotted as a percentage of the $R-R^{\prime}$ interval obtained as an average from 9 cardiac cycle pairs. Note that the correlation values have a narrower peak than the corresponding graph from subject 1 shown in Figure 1E. LV indicates left ventricle. 


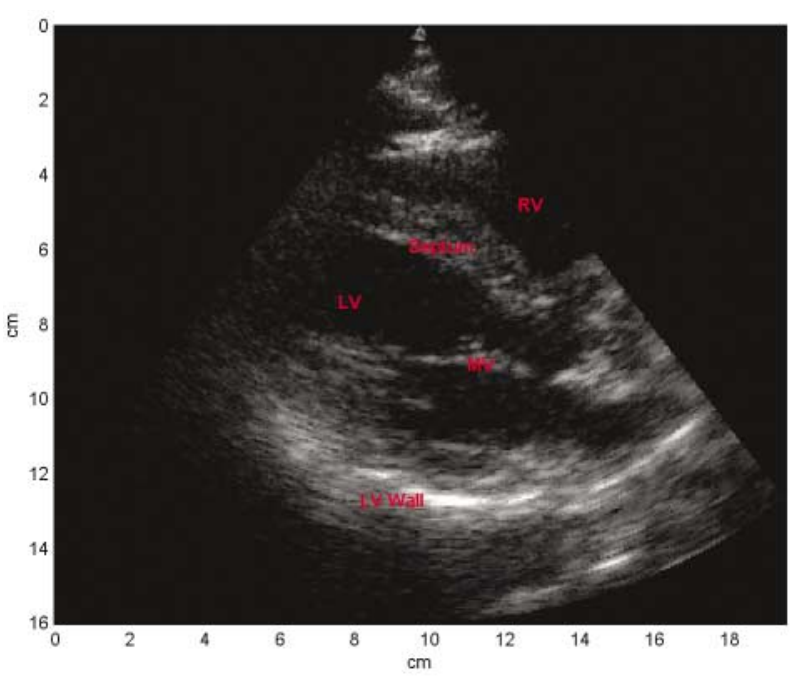

A

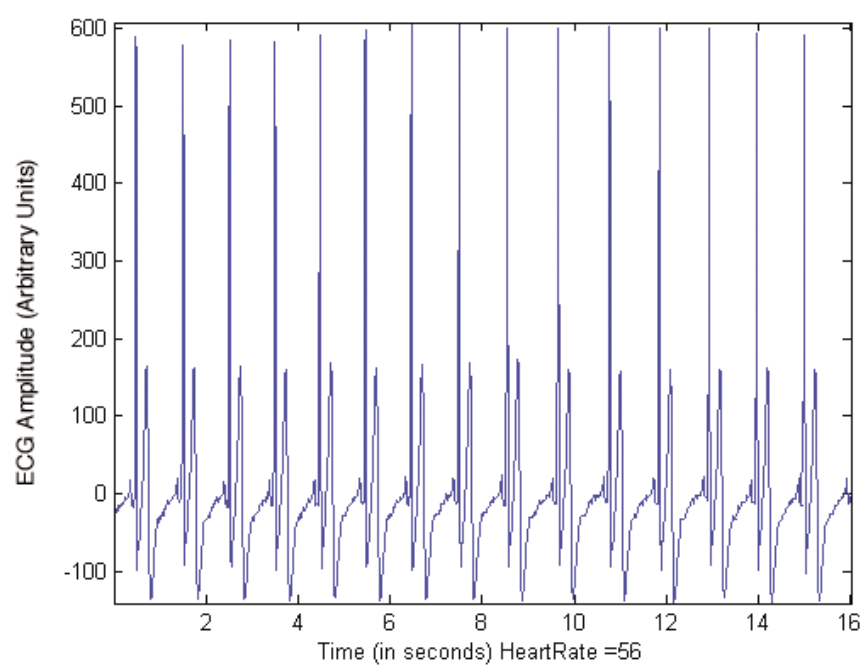

C

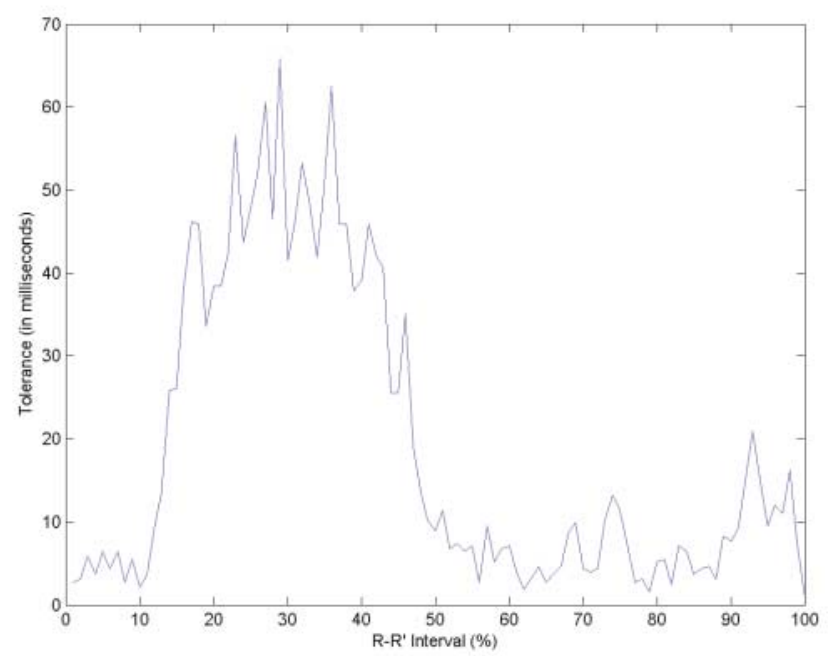

$\mathbf{E}$

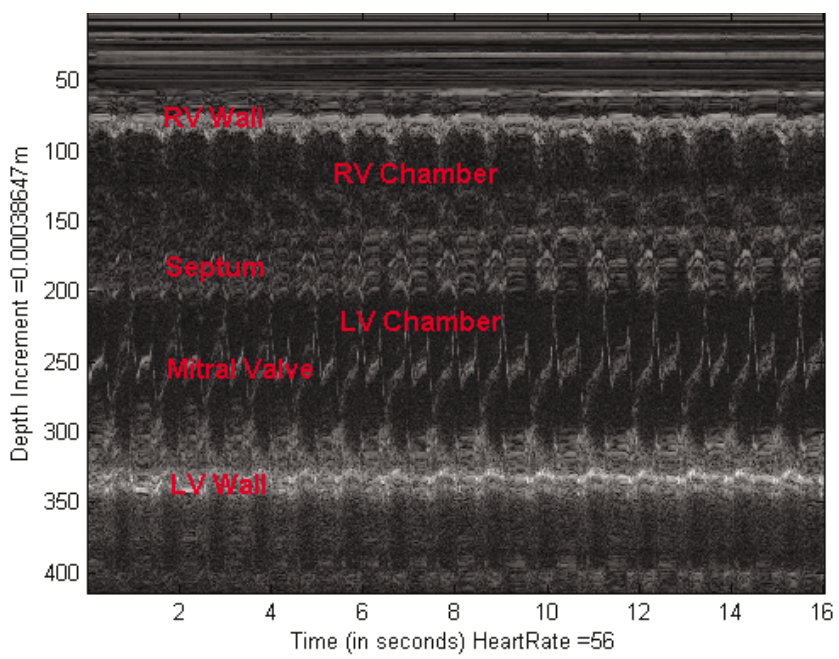

B

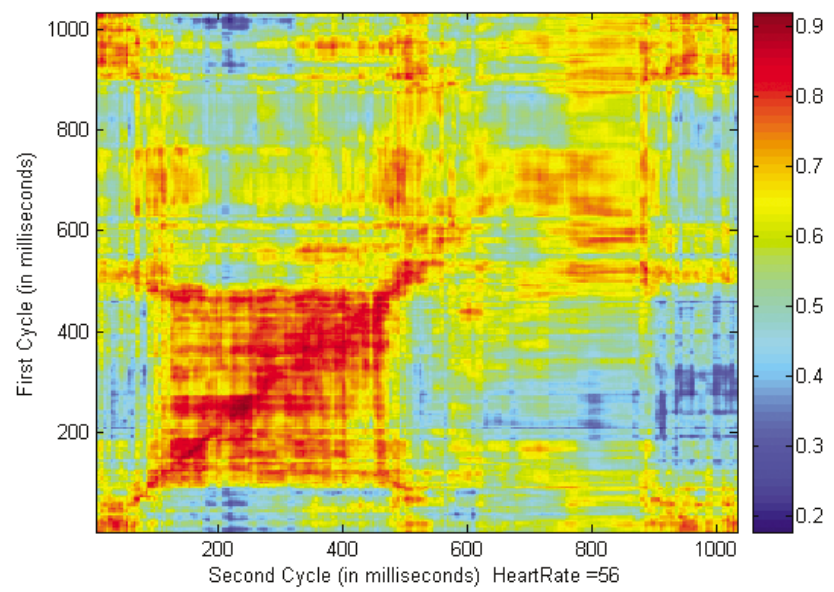

D

Figure 3. Subject 2, data obtained in the long axis left parasternal view near the base. A, B-mode image. B, Corresponding M-mode data. C, Corresponding ECG. D, Correlation plot between a randomly selected cycle and a subsequent cycle. E, Tolerance values plotted as a percentage of the R- $R^{\prime}$ interval obtained as an average from 9 cardiac cycle pairs. Note that the correlation values have a broad peak that appears earlier in the cardiac cycle than the corresponding graph from subject 2 obtained in the long axis left parasternal view near the apex (shown in Figure 2E). LV indicates left ventricle; $M V$, mitral valve; and $R V$, right ventricle. 
reconstructions from different phases of the cardiac cycle in the same patient can result in completely different estimations of coronary risk, which range between low risk and increased risk; even more surprisingly, nearly $63 \%$ of patients could be assigned to more than 1 risk group, and $10 \%$ could be assigned to 3 risk groups. When examining the width of the tolerance peaks in Figure 4, one can see the potential effect of selecting slices from different points in the cardiac cycle for reconstructions.

In addition, using our method, one could theoretically gate during any portion of the heart cycle. One would only need to estimate the correlation matches of the ultrasonic signals for sets of projections. For instance, if one wanted to reconstruct during midsystole, it would just require more heartbeats to acquire the data. That is, if the heart is only sufficiently correlated during 4 milliseconds of each beat, it would take 50 heartbeats to acquire sufficient data if a 200-millisecond total time is required for a minimum reconstruction.

Ultimately, we think that, given the current state of the art in digital signal- and array-processing technologies, it should be possible to implement the cross-correlation sequence in real time, thus obtaining a real-time triggering mechanism that would be independent of ECG. Thus, a sonographically based gating method could potentially be applied prospectively. Finally, sonographic gating could be used to improve retrospective gating by saving the time-dependent sonographic images along with the associated projection data. The correlations would then determine which projections would be used together to produce the best reconstructions.

Of course, the presence of an ultrasound transducer could cause a streak artifact within a CT scanner or a susceptibility artifact in an MR scanner. Interference effects may also occur with MRI requiring shielding of the ultrasound probe wires, although it has already been shown by Rubin et $\mathrm{al}^{4}$ that Doppler ultrasound scanning can be used to gate cardiac MRI scans. The technique we have described, however, could be used with a very simple, single-element transducer, which presumably could be designed so that it has very little effect on the CT or MR images, similar to ECG electrodes. It is not inconceivable that such a simple probe can be affixed to the chest wall with tape or adhesive, much like

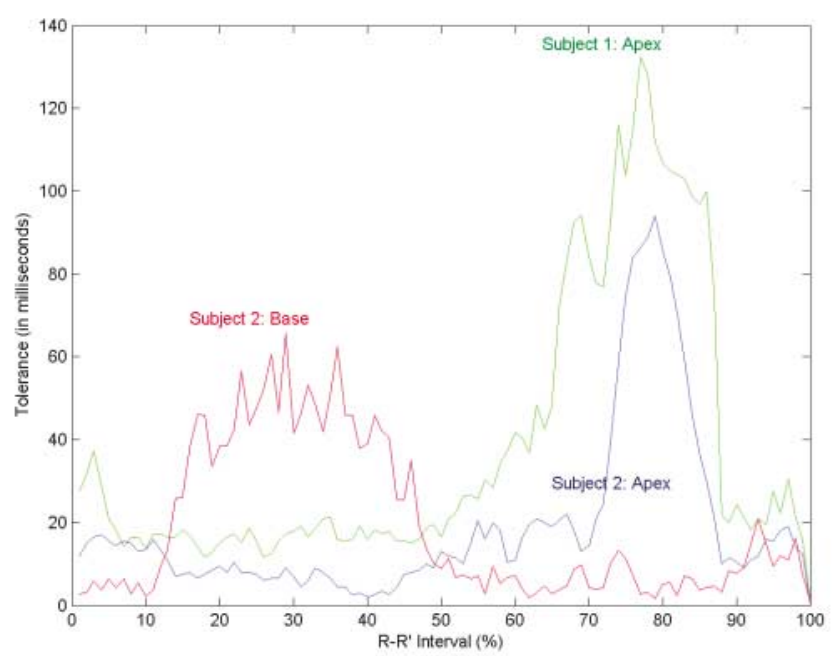

Figure 4. Tolerance or quiescent period versus the $R-R^{\prime}$ interval (percent). These are curves consolidated from Figures $1 \mathrm{E}, 2 \mathrm{E}$, and $3 \mathrm{E}$. The tolerance at the apex is shown for both subjects 1 and 2 and at the base for subject 2 . The base has a quiescent period at around $30 \%$, and the apex has a quiescent period later in the cycle; this period starts slightly earlier for subject 1 and also lasts longer than for subject 2 .

ECG electrodes, and thus not require an operator to hold the probe while the patient is being scanned. Furthermore, we take advantage of the fact that correlation processing is very resistant to random noise in data, making "poor image quality" less of an issue. In fact, it is conceivable that one could gate off unfocused 3D sonographic samplings of the beating heart. Any repetitive time signature in the backscattered echoes would suffice for gating, even if no image were produced. These issues will need to be investigated before the implementation of a real-time approach.

There are some limitations to our study. The chief limitation is that, in this preliminary study, we did not evaluate the final CT or MR images that would result from an implementation of our approach. Directly combining our gating scheme with CT data will require access to raw projection data for retrospective reconstructions or a direct interface with the CT gantry for prospective gating. This will require an arrangement with a CT manufacturer, which we do not as yet have but are in the process of trying to arrange. A second issue is that we performed analysis on M-mode data only because of the preliminary nature of this study. However, the same type of analysis could be performed with 
2D sonographic scans or unfocused 3D timedependent data as mentioned above, although 2D and 3D methods have the problem of reduced temporal resolution and may or may not be useful. A further criticism is that we have presented data from only 2 patients. This is not really a limitation because our goal was not to show that a particular $\mathrm{R}-\mathrm{R}^{\prime}$ interval can be used to trigger CT optimally across the population. Instead, our goal was to show that there are variations between subjects and the portions of the heart being imaged. Because our goal was to prove inconsistencies, we needed only 1 counterexample. Furthermore, the 2 subjects who participated in this study were healthy subjects; the variations are likely to be even higher in patients with arrhythmias. We also arbitrarily chose correlation coefficients of greater than 0.8 as our threshold. If higher correlations are necessary for better reconstructions, the threshold would be adjusted to accommodate them. If lower correlation thresholds are sufficient for certain purposes, they could be used, thus decreasing the duration of the examination.

In conclusion, we have presented preliminary data suggesting that cardiac CT or MRI could be gated with real-time sonography to monitor cardiac wall motion. The method has the potential to overcome some of the limitations of ECG and thus improve the quality and consistency of cardiac and coronary artery cross-sectional imaging.

\section{References}

1. Achenbach S, Daniel WG. Noninvasive coronary angiography: an acceptable alternative? N Engl J Med 2001; 345:1909-1910.

2. Budoff MJ, Achenbach S, Duerinckx A. Clinical utility of computed tomography and magnetic resonance techniques for noninvasive coronary angiography. J Am Coll Cardiol 2003; 42:1867-1878.

3. Desjardins B, Kazerooni EA. ECG-gated cardiac CT. AJR Am J Roentgenol 2004; 182:993-1010.

4. Rubin JM, Fowlkes JB, Prince MR, Rhee RT, Chenevert TL. Doppler US gating of cardiac MR imaging. Acad Radiol 2000; 7:1116-1122.

5. Schlosser T, Hunold P, Schmermund A, et al. Coronary artery calcium score: influence of reconstruction interval at 16-detector row CT with retrospective electrocardiographic gating. Radiology 2004; 233:586-589.
6. Agatston AS, Janowitz WR, Hildner FJ, Zusmer NR, Viamonte MJ, Detrano R. Quantification of coronary artery calcium using ultrafast computed tomography. J Am Coll Cardiol 1990; 15:827-832. 